\title{
Extent of resection and radiotherapy in GBM: A 1973 to 2007 surveillance, epidemiology and end results analysis of 21,783 patients
}

\author{
PASCAL O. ZINN ${ }^{1}$, RIVKA R. COLEN ${ }^{2}$, EKKEHARD M. KASPER ${ }^{3}$ and JAN-KARL BURKHARDT ${ }^{4,5}$ \\ ${ }^{1}$ Department of Neurosurgery, Baylor College of Medicine, Houston, TX; ${ }^{2}$ Department of Radiology, \\ M.D. Anderson Cancer Center, University of Texas, Houston, TX; ${ }^{3}$ Department of Surgery, Division of \\ Neurosurgery, Beth Israel Deaconess Medical Center, Harvard Medical School, Boston, MA, USA; ${ }^{4}$ Department of \\ Neurosurgery, University Hospital, Zurich, Switzerland; ${ }^{5}$ Department of Neurological Surgery, Weill Cornell \\ Brain Tumor Center, Presbyterian Hospital, Weill Cornell Medical College, New York, NY, USA
}

Received May 9, 2012; Accepted July 16, 2012

DOI: $10.3892 /$ ijo.2013.1770

\begin{abstract}
Surgery, radiation and chemotherapy are the standard of care for GBM patients, however, the impact of extent of resection (EOR) and radiotherapy (RT) on patient survival across age groups has not been established. Therefore, we present the current largest study on EOR and RT in GBM over the past three decades. Using the population based Surveillance, Epidemiology and End Results (SEER) registry, we identified a total of 21,783 GBM patients (1973-2007). Survival analysis based on EOR and RT was performed by means of factor analysis, Kaplan-Meier survival and Cox proportional hazard's ratio. Age, RT and EOR were highly prognostic $(\mathrm{p}<0.00001)$. Combined gross total resection (GTR) and RT showed the longest median survival (11 months) compared to subtotal resection (STR) and RT (9 months). Survival times after monotherapy with RT, GTR and STR were 5, 3 and 2 months, respectively. Patients without therapy showed a median survival of 1 month. RT and GTR demonstrated highest median survival. Interestingly, survival advantage of GTR versus STR amounted to only 1-2 months. Monotherapy (GTR, STR or RT) showed a significantly lower survival rate compared to combination therapies. RT alone yielded significantly better survival compared to any resective approach. Relative to overall age-specific median survival, elderly patients still reasonably benefit from RT alone. However, across all age groups multimodality treatment with surgery and RT continues to provide the largest survival benefit compared to either treatment alone and, thus, should be pursued whenever feasible.
\end{abstract}

Correspondence to: Dr Pascal O. Zinn, Department of Neurosurgery, Baylor College of Medicine, 1 Baylor Plaza, Houston, TX, USA

E-mail: zinn@bcm.edu

Key words: glioblastoma multiforme, extent of resection, radiotherapy, survival, SEER, epidemiology

\section{Introduction}

Malignant gliomas, including the most common and fatal form glioblastoma multiforme (GBM), remain a challenge to treat (1-14). In the United States alone, more than 10,000 patients per year are newly diagnosed with GBM (15). Despite ongoing trials, the best currently available multimodal treatment approaches include surgical resection followed by adjuvant radiation and temozolomide treatment (RT/TMZ), resulting in a low median overall survival (OS) ranging from 12.2-15.9 months $(5,9-11,16)$. Previous studies have shown that survival rates are significantly influenced by certain clinical and molecular factors $(2,3,6,8,17-21)$. Of those, surgical gross total resection (GTR), which is defined as a complete removal of the contrast enhancing portion of the tumor as measured on postoperative MRI by volumetric analysis, is one of the important independent predictors of patient survival $(5,9-11,13)$. Stupp et al showed a median survival rate of 18.8 months after GTR versus 13.5 months after subtotal resection (STR) versus 9.4 months after biopsy alone (13). These findings were confirmed by McGirt et al who in a large series of 700 GBM patients demonstrated a survival benefit of approximately 5-6 months after primary and secondary GTR, when compared to STR (9). A more recent study by Sanai et al demonstrated that pursuing a more aggressive EOR results in increased survival, especially in patients with a higher degree of tumor removal. Thus, STR of $78 \%$ or more still yielded a significant survival benefit in patients afflicted with GBM (11).

Beyond the EOR, the postoperative adjuvant treatment regimen is most relevant in determining patient outcome and multiple studies indicate that a combination of radiochemotherapy and especially RT/TMZ further improves patient survival $(3,13,14)$. However, the majority of previous studies have been comparing survival and EOR in GBM patients admitted to a neurosurgical service and receiving RT/TMZ $(11,13,14)$ and hence only examined a selected group of GBM patients. The recently published studies by Koshy et al and Johnson et al were the first groups comparing survival of GBM patients from the Surveillance, Epidemiology and 
End Results (SEER) program before and during the TMZ era and showed a significant survival benefit in TMZ treated patients $(22,23)$. In these studies, however, only patients from the last decade (2000-2008) were included for analysis, without subgroup analysis with regard to RT and EOR. In our here presented population-based study, which spans over the past three decades, we provide a comprehensive analysis of GBM patient data to demonstrate the overall and age-specific impact of RT compared to EOR and its relevance for prognosis.

\section{Materials and methods}

SEER and data extraction. The Surveillance, Epidemiology and End Results (SEER) program of the National Cancer Institute, established in 1973, collects incidence and survival data for patients with malignant tumors from selected population-based cancer registries across the US. Since this data set is population-based, it captures a defined geographical area with a demographically well-defined population representing $28 \%$ of the US population; regions were selected by NCI for inclusion into SEER for their ability to operate a population-based cancer registry and for their diverse population subgroups. Data for this study were downloaded from the SEER publicuse homepage (http://seer.cancer.gov/, NCI, Bethesda, MD) and were converted to Microsoft Access and Excel databases for data extraction and further analysis.

Patient population and variable collection. A total of 21,783 patients diagnosed with GBM (SEER code 9440/ICD-O-3) between 1973 and 2007 was identified and included for analysis in this study. Of those, the following patient variables were analyzed: year of diagnosis, age at diagnosis, gender, race/ethnicity, laterality of lesion, number of primaries (single versus multiple lesions), radiation treatment (RT), extent of resection (EOR) and survival. For comparative analysis the maximum common amount of available patients per set of variables was chosen.

In a second step patients were binned in 10-year age intervals $(<20,20-30,30-40,40-50,50-60,60-70,70-80$ and $>80$ years $)$ and data were analyzed for overall and treatment specific survival (Table II).

Statistical analysis. Factor analysis and Cox proportional hazards ratio were used to determine the variables most closely correlated to survival and most relevant for prognosis. Overall and treatment specific survival was computed using the Kaplan-Meier method and the log-rank test. Respective SEER data use policies have been adhered to for this study. The statistical analysis was performed using Microsoft Excel and Access 2010, JMP Pro 9.01 (SAS, Cary, NC) statistical software packages.

\section{Results}

Demographics. The mean onset age of GBM in the analyzed patient cohort of 21,783 individuals was 61.5 years (male, 60.5 years; female, 63 years) and the gender distribution was $57 \%$ male $(\mathrm{N}=12,447)$ and $43 \%$ female $(\mathrm{N}=9336)$. The ethnicity in the SEER patient data set with GBM (SEER code 9440/ICD-O-3) was predominantly Caucasian white with $92 \%(\mathrm{~N}=20,057)$ followed by African American
0.05\% ( $\mathrm{N}=966$ ), similar ethnical GBM data were previously reported $(24,25)$.

Cluster correlation and survival. The median OS of all patients was 6 months for individuals included in this study (women, 6 months; men, 7 months) (Fig. 1a). The age-adjusted survival development over time shows a marked increase of overall median survival after 1997 (Fig. 1b). In the factor analysis the best negative correlation to survival was observed for age, the best positive correlation for EOR and RT (Fig. 2a and b). The most significant $(\mathrm{p}<0.00001)$ prognostic survival variables were: RT, age and EOR and in the proportional hazard's ratio test, these 3 variables were found to be significant independent predictors for patient survival (Table I).

With regard to EOR, the median OS reported for GTR patient was 10 months compared to 8 months for STR or 5 months for biopsy only (all p<0.00001) (Fig. 3). Kaplan-Meier analysis of patients with and without RT reported a survival benefit of approximately 7 months for patients in the RT group ( $<<0.00001$; Fig. 4). Because both RT and EOR were significant and independent prognostic variables, we further subcategorized the patient cohort as follows: i) GTR/RT, ii) GTR alone, iii) STR/RT, iv) STR alone, v) RT alone and vi) no surgery/no RT. In this subset analysis, patients with neither surgery nor RT showed a median survival of only 1 month. Likewise STR survival without RT demonstrated a low survival (2 months) and GTR without RT showed a relatively low median survival of 3 months. Interestingly, patients treated with RT without any surgical intervention had a higher median survival (5 months) than patients treated with any surgical monotherapy. When surgery and RT was combined, median survival markedly increased for both GTR/RT (11 months) and STR/RT (9 months) (Fig. 5, Table II).

Age distribution and survival variables. Analysis of patients binned into 10-year age subgroups demonstrates that patients receiving neither surgical treatment nor RT showed a median survival of only 1 month across all age groups. Furthermore, patients undergoing STR without RT showed a survival advantage of 1 month when 30 years and older, whilst STR in patients below age 30 showed an average of more than 5 months of increased survival when compared to patient receiving no treatment at all.

GTR alone improved survival when compared to both no treatment and STR in all age groups. However, relative to the age specific overall median survival, GTR alone results in below average survival in patients younger than 70 years (Table II, Fig. 6). RT, when employed as a monotherapy, is superior to both GTR and STR alone across all age groups. But it results in significantly decreased median survival compared to overall age-specific survival, except in elderly patients above 70 years of age. Either combined GTR or STR with adjuvant RT yielded the highest median survival across all examined age groups. With increasing age, the relative survival advantage compared with the overall age specific median survival advantage increases with more aggressive therapy (Fig. 6). When comparing median survival times in patients undergoing STR or GTR monotherapies across age intervals, less distinct survival differences are demonstrated 
Table I. Effect likelihood ratio table: EOR, RT and age are significant independent predictor for patient survival in GBM.

\begin{tabular}{lcccc}
\hline Variable & Nparm & DF & Chi-square & p-value \\
\hline Extent of resection & 4 & 4 & 897.256437 & $<0.00001$ \\
Radiation treatment (RT) & 3 & 3 & $2,739.97669$ & $<0.00001$ \\
Age & 1 & 1 & $1,015.88061$ & $<0.00001$ \\
\hline
\end{tabular}

a)

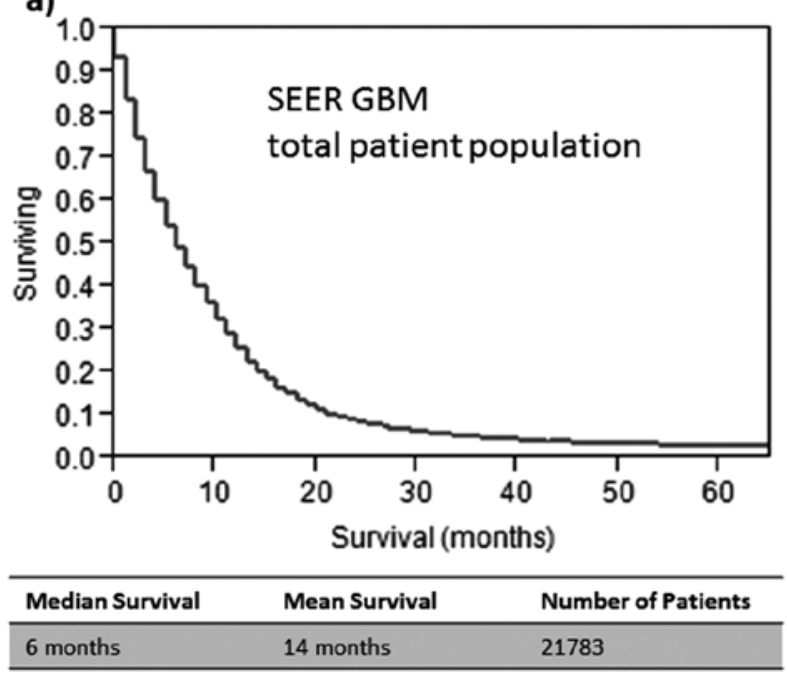

b)

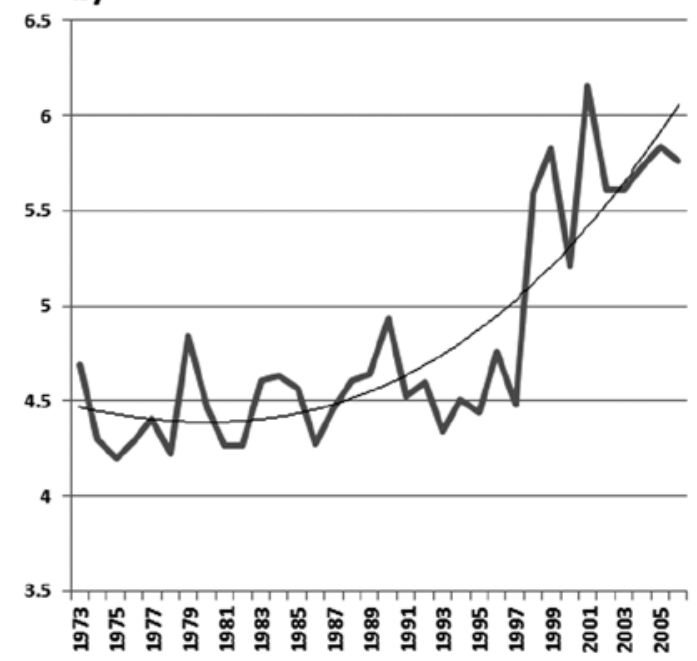

Figure 1. (A) Kaplan-Meier plot, SEER overall 21,783 GBM patient median survival and (B) yearly median survival development over time (y-axis, median survival in months; $\mathrm{X}$-axis, timeline in years).

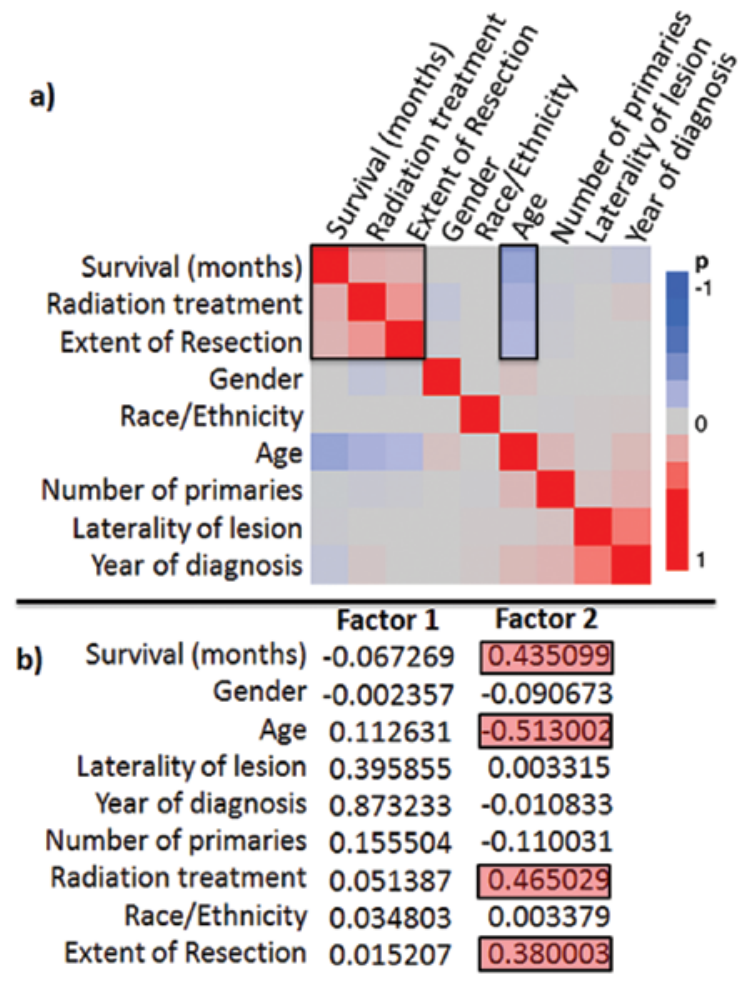

Figure 2. (A) Factor analysis with nine glioblastoma patient variables and (B) cluster correlation heatmap showing survival, RT and EOR clusters. Age is negatively correlated.

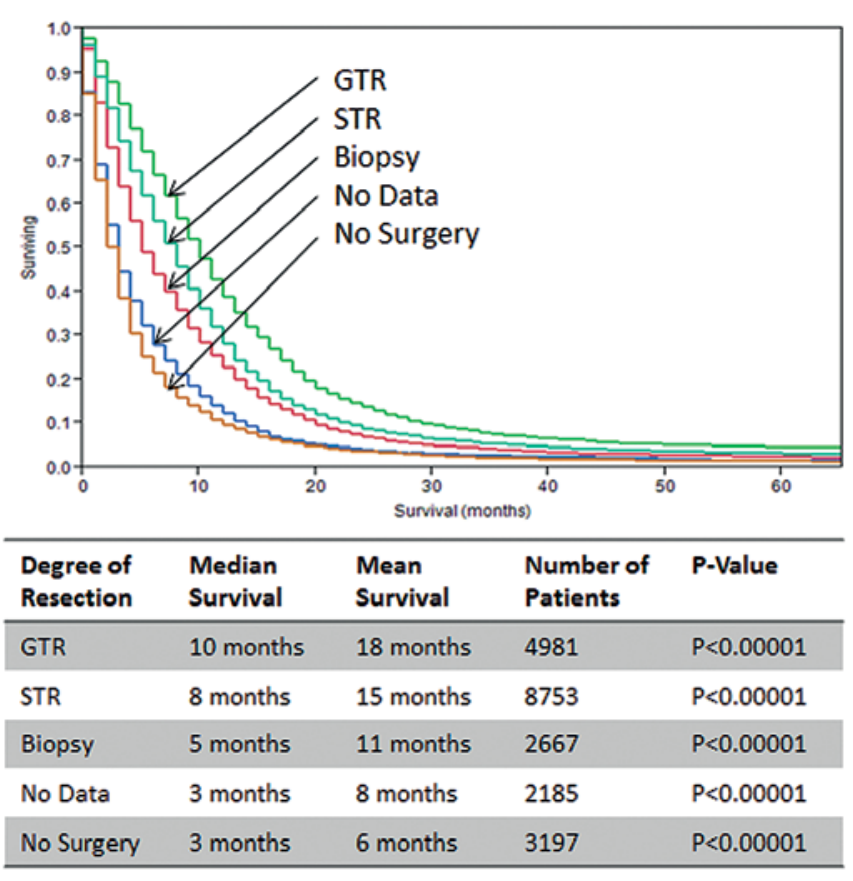

Figure 3. Kaplan-Meier plot, EOR with a comparison of GTR, STR, biopsy, no surgery and no data (2,185 patients). 


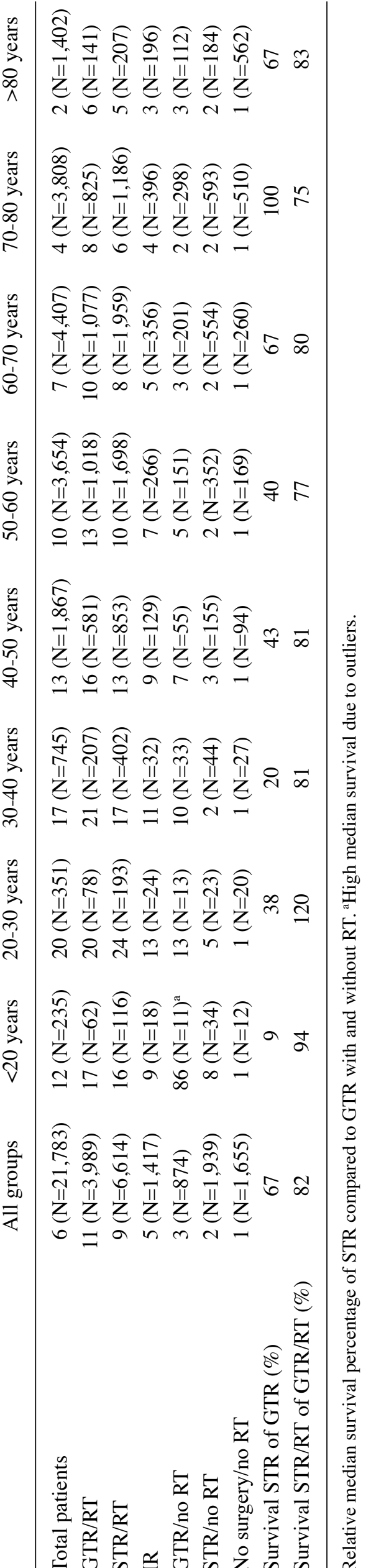

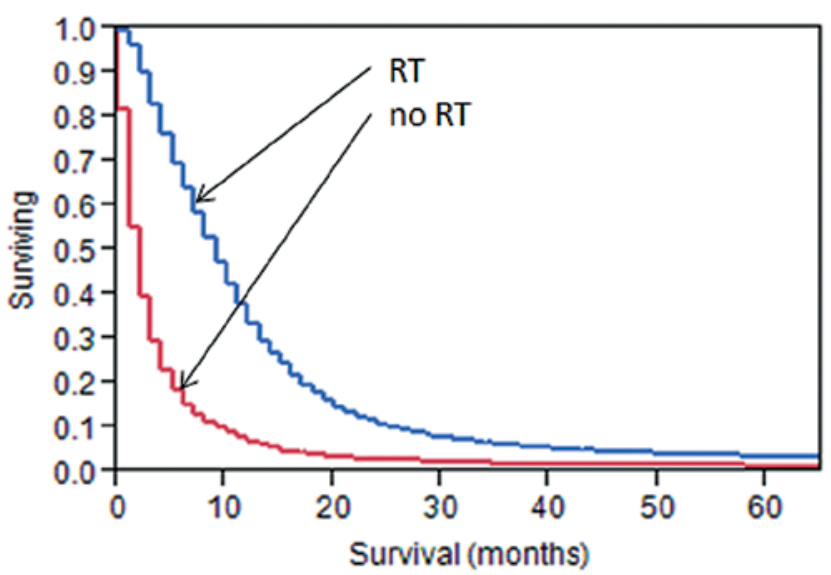

\begin{tabular}{lllll}
\hline $\begin{array}{l}\text { Degree of } \\
\text { Resection }\end{array}$ & $\begin{array}{l}\text { Median } \\
\text { Survival }\end{array}$ & $\begin{array}{l}\text { Mean } \\
\text { Survival }\end{array}$ & $\begin{array}{l}\text { Number of } \\
\text { Patients }\end{array}$ & P-Value \\
\hline $\begin{array}{l}\text { Radiation } \\
\text { Therapy (RT) }\end{array}$ & 9 months & 17.5 months & 15008 & P $<0.00001$ \\
$\begin{array}{l}\text { No Radiation } \\
\text { Therapy (RT) }\end{array}$ & 2 months & 6.5 months & 6129 & P $<0.00001$ \\
\hline
\end{tabular}

Figure 4. Kaplan-Meier plot, comparison of patients receiving RT versus no RT. In 646 patients no data were available.

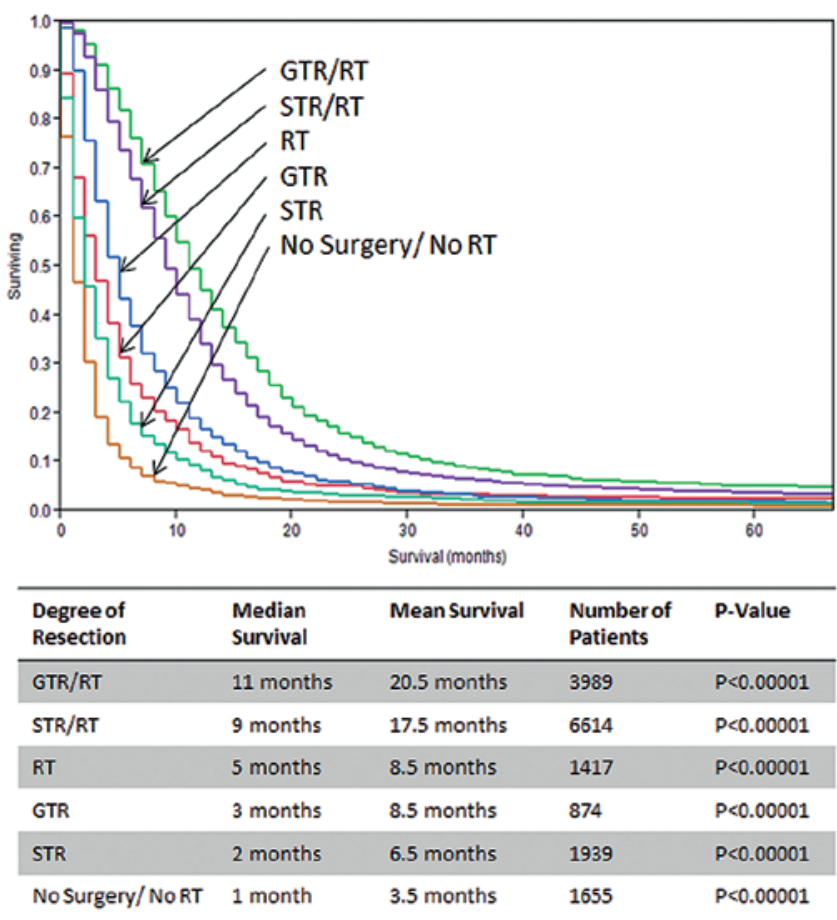

Figure 5. Kaplan-Meier plot, GTR, STR and no surgical intervention paired with and without RT resulting in the following groups: GTR/RT, STR/RT, RT, GTR, STR, no surgery/no RT. In 5,295 patients no data were available.

with increasing age. Of note, in the setting of adjuvant RT; STR and GTR yielded very similar effects on survival in the age groups below 30 years (STR/GTR, 94-120\%). This relative survival contribution remained stable at $75-83 \%$ when examined across advancing age groups (Table II). 


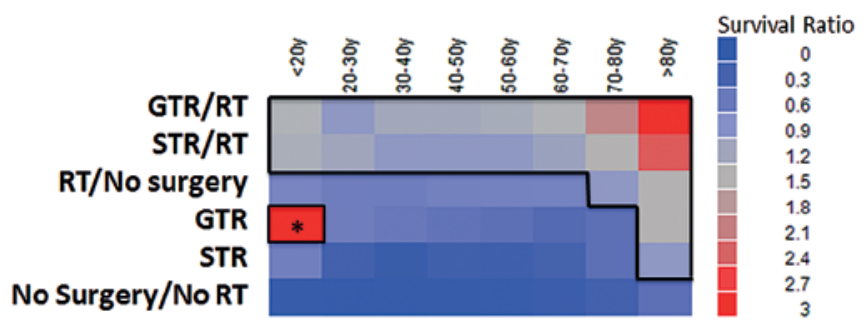

Relative median survival ratio (fold change) across age groups compared to age specific median survival

* small patient number

- above median survival

Figure 6. Relative median survival advantage across age groups compared to overall median survival. Red, higher than median survival compared to overall age specific median survival; blue, lower than median survival compared to overall age specific median survival.

\section{Discussion}

In this study, we identified RT, age and EOR to be significant and independent prognostic factors in a cohort of 21,783 GBM patients. From the variables selected for analysis, RT, age and EOR were most closely correlated with outcome as reflected in survival times (Fig. 2). These three variables were all highly prognostic $(\mathrm{p}<0.00001)$ as well as independent predictors for survival in the Cox proportional hazards ratio (Table I). The fact that age continues to be a strong predictor of survival is concordant with previously published literature. Among various other age-related general factors, this is thought to reflect that GBM in older patients are most commonly of the primary (de novo) type. This type is more aggressive, invasive, and has a different genomic and molecular microenvironment $(26,27)$ when compared to secondary GBM seen more commonly in younger patients.

With regard to the relevance of EOR, the scientific debate is ongoing, but our study clearly demonstrates an overall median survival advantage, which is significantly higher for GTR (10 months) when compared to STR (8 months) or biopsy (5 months) ( $<<0.00001$; Fig. 3). The survival benefit of GTR compared to STR is concordant with the literature; however, studies disagree on a threshold of resection leading to a significant survival advantage $(10,11,13)$.

Nevertheless, our analysis indicates that any reasonable surgical resection improves survival by $8-10$ months in the setting of adjuvant RT. This is of great clinical significance and challenges the previous widely held belief, which suggests that only GTR or near total resections of greater than $98 \%$ of tumor volume offers a significant survival benefit (5).

A recent study by Sanai et al (11), similarly challenged this doctrine by demonstrating that STR of more than $78 \%$ of tumor volume already benefitted the patient with respect to posttreatment survival. Our results as well as those from the latter study suggest that EOR is a significant predictor of survival and offers hope to those patients in whom GTR is not achievable. Surgical resection in absence of adjuvant RT improves median survival by 1-2 months only and thus confers a minimal advantage compared to patients who did neither undergo resection nor
RT. Conversely, RT alone resulted in a 2-3 months prolonged survival period when compared to monotherapy with GTR or STR (Fig. 5). The relative shorter survival times in either the surgery alone (2-3 months) or RT alone groups (5 months) compared to concurrent treatment (9-11 months) suggests a beneficial treatment synergy, but may also reflect the fact that healthier patients are more likely to receive more comprehensive therapy.

The presented median OS of 6 months derived from the whole SEER GBM patient cohort is relatively low, when compared to other published studies presenting a median OS range of 12.2-15.9 months (5,9-11). This might be due to the fact that these data sets, unlike the SEER, for the most part represent a highly preselected group of patients (e.g. admitted to an expert neurosurgical oncology service, also receiving adjuvant TMZ/RT at different intervals) (9-11).

The strength of this study clearly is the dataset spanning more than 3 decades with the advantage of not being influenced by selection biases associated with treatment and referral patterns seen in many of the previous studies. Furthermore, a clear upward trend for survival (Fig. 1b) can be seen starting in 1997, this may coincide with the introduction of novel chemotherapeutic agents, refined irradiation protocols, and advanced surgical techniques $(5,11,28,29)$. These results are also in agreement to the recently published studies comparing the adjuvant era before and after introduction of temozolomide therapy $(22,23)$. Both studies showed a significant patient survival benefit after the presented EORTC/NCIC trial in 2004. Regardless of this study's limitations inherent to SEER (such as lack of adjuvant chemotherapy and quantitative EOR data, as well as less standardized data collection/documentation compared to smaller single center studies) the strength of our study lies within the large scale population-based approach.

We clearly corroborated data that patients, who receive postoperative RT survived 7 months longer than non-radiated patients ( $<<0.00001$; Fig. 4). It was also obvious that RT without surgery resulted in consistently better survival than survival in those patients who were treated by surgery alone, whether GTR or STR (Fig. 5). This difference was confirmed across all age groups (Table II). This is of great clinical significance since elderly patients or those with surgical contraindications can benefit from treatment, even when receiving RT alone. RT as a suitable treatment modality for GBM patients was analyzed in the elderly before and our results confirm the findings of these studies with a significant survival benefit of patients receiving RT compared to patients who do not $(12,30,31)$. However, the more aggressive the treatment regimen, the higher the relative survival gain compared to the age specific median survival and this is seen particularly in the elderly patient (Fig. 6). Thus, we were able to show that even patients of 80 years and older, if eligible, should undergo aggressive treatment since this can result in prolonged survival. In general, our study demonstrates that a comprehensive treatment regimen is of utmost importance to prolong survival in GBM patients across all age groups.

Age specific analysis suggested GTR to be superior to STR particularly in younger patients. Interestingly, in patients below 30 years of age this advantage was lost when RT was added to the treatment regimen, stressing the importance of adjuvant RT. 


\section{Acknowledgements}

Surveillance Research Program, National Cancer Institute SEER*Stat software (seer.cancer.gov/seerstat).

\section{References}

1. Hess KR, Broglio KR and Bondy ML: Adult glioma incidence trends in the United States, 1977-2000. Cancer 101: 2293-2299, 2004.

2. Buckner JC: Factors influencing survival in high-grade gliomas. Semin Oncol 30: 10-14, 2003.

3. Hegi ME, Diserens AC, Gorlia T, et al: MGMT gene silencing and benefit from temozolomide in glioblastoma. N Engl J Med 352: 997-1003, 2005.

4. Iwamoto FM, Reiner AS, Nayak L, Panageas KS, Elkin EB and Abrey LE: Prognosis and patterns of care in elderly patients with glioma. Cancer 115: 5534-5540, 2009.

5. Lacroix M, Abi-Said D, Fourney DR, et al: A multivariate analysis of 416 patients with glioblastoma multiforme: prognosis, extent of resection, and survival. J Neurosurg 95: 190-198, 2001.

6. Lamborn KR, Chang SM and Prados MD: Prognostic factors for survival of patients with glioblastoma: recursive partitioning analysis. Neuro Oncol 6: 227-235, 2004.

7. Lefranc F, Rynkowski M, DeWitte O and Kiss R: Present and potential future adjuvant issues in high-grade astrocytic glioma treatment. Adv Tech Stand Neurosurg 34: 3-35, 2009.

8. Li SW, Qiu XG, Chen BS, et al: Prognostic factors influencing clinical outcomes of glioblastoma multiforme. Chin Med J (Engl) 122: 1245-1249, 2009.

9. McGirt MJ, Chaichana KL, Gathinji M, et al: Independent association of extent of resection with survival in patients with malignant brain astrocytoma. J Neurosurg 110: 156-162, 2009.

10. Sanai N and Berger MS: Glioma extent of resection and it impact on patient outcome. Neurosurgery 62: 753-764, 2008.

11. Sanai N, Polley MY, McDermott MW, Parsa AT and Berger MS An extent of resection threshold for newly diagnosed glioblastomas. J Neurosurg 115: 3-8, 2011.

12. Scott J, Tsai YY, Chinnaiyan P and Yu HH: Effectiveness of radiotherapy for elderly patients with glioblastoma. Int J Radiat Oncol Biol Phys 81: 206-210, 2011.

13. Stupp R, Hegi ME, Mason WP, et al: Effects of radiotherapy with concomitant and adjuvant temozolomide versus radiotherapy alone on survival in glioblastoma in a randomised phase III study: 5-year analysis of the EORTC-NCIC trial. Lancet Oncol 10: 459-466, 2009.

14. Stupp R, Mason WP, van den Bent MJ, et al: Radiotherapy plus concomitant and adjuvant temozolomide for glioblastoma. N Engl J Med 352: 987-996, 2005.
15. CBTRUS: Central Brain Tumor registry of the United States. http://www.cbtrus.org/ 2008

16. Zinn PO, Sathyan P, Mahajan B, Bruyere J, Hegi M, Majumder S, Colen RR: A novel volume-age-KPS (VAK) glioblastoma classification identifies a prognostic cognate microRNA-gene signature. PLoS One 7: e41522, 2012.

17. Marko NF, Toms SA, Barnett GH and Weil R: Genomic expression patterns distinguish long-term from short-term glioblastoma survivors: a preliminary feasibility study. Genomics 91: 395-406, 2008.

18. Phillips HS, Kharbanda S, Chen R, et al: Molecular subclasses of high-grade glioma predict prognosis, delineate a pattern of disease progression, and resemble stages in neurogenesis. Cancer Cell 9: 157-173, 2006.

19. Colman H, Zhang L, Sulman EP, et al: A multigene predictor of outcome in glioblastoma. Neuro Oncol 12: 49-57, 2010.

20. Cooper LA, Gutman DA, Long Q, et al: The proneural molecular signature is enriched in oligodendrogliomas and predicts improved survival among diffuse gliomas. PLoS One 5: e12548, 2010

21. Zinn PO, Majadan B, Sathyan P, et al: Radiogenomic mapping of edema/cellular invasion MRI-phenotypes in glioblastoma multiforme. PLoS One 6: e25451, 2011.

22. Johnson DR and O'Neill BP: Glioblastoma survival in the United States before and during the temozolomide era. J Neurooncol 107, 359-364, 2012.

23. Koshy M, Villano JL, Dolecek TA, et al: Improved survival time trends for glioblastoma using the SEER 17 populationbased registries. J Neurooncol 107: 207-212, 2012.

24. Barnholtz-Sloan JS, Sloan AE and Schwartz AG: Racial differences in survival after diagnosis with primary malignant brain tumor. Cancer 98: 603-609, 2003.

25. Barnholtz-Sloan JS, Maldonado JL, Williams VL, et al: Racial/ethnic differences in survival among elderly patients with a primary glioblastoma. J Neurooncol 85: 171-180, 2007.

26. Ang C, Guiot MC, Ramanakumar AV, Roberge D and Kavan P: Clinical significance of molecular biomarkers in glioblastoma. Can J Neurol Sci 37: 625-630, 2010.

27. Kanu OO, Hughes B, Di C, et al: Glioblastoma multiforme oncogenomics and signaling pathways. Clin Med Oncol 3: 39-52, 2009.

28. Chang JE, Khuntia D, Robins HI and Mehta MP: Radiotherapy and radiosensitizers in the treatment of glioblastoma multiforme. Clin Adv Hematol Oncol 5: 894-915, 2007.

29. Clarke J, Butowski N and Chang S: Recent advances in therapy for glioblastoma. Arch Neurol 67: 279-283, 2010.

30. Keime-Guibert F, Chinot O, Taillandier L, et al: Radiotherapy for glioblastoma in the elderly. N Engl J Med 356: 1527-1535, 2007.

31. Lai R, Hershman DL, Doan T and Neugut AI: The timing of cranial radiation in elderly patients with newly diagnosed glioblastoma multiforme. Neuro Oncol 12: 190-198, 2010. 